\title{
Classification of the Systems Used in Surface Electromyographic Signal Detection according to the Degree of Isotropy
}

\author{
Noureddine Messaoudi, ${ }^{*, *}, \#$ Raïs El'hadi BekKa, ${ }^{* *}$ Samia BelKacem ${ }^{*}$
}

\begin{abstract}
Surface electromyographic (EMG) signals are known to be strongly influenced by anatomical, physiological and detection system parameters. Among the detection system parameters, we are interested in the effect of muscle fiber inclination on the electrode arrangement. The purpose of this study was to determine the best and the worst orientation of the electrodes arranged in nine detection systems relative to the muscle fiber direction and also to classify the investigated systems according to their degree of isotropy. The study was based on simulated surface EMG (sEMG) signals generated in a cylindrical multilayer volume conductor. The orientation of electrodes with respect to the fiber direction was defined by the fiber inclination angle (FIA). For each detection system, the mean power (MP) of the simulated signals was computed at different FIAs and used as a basis for evaluating the effect of muscle fiber inclination. We showed that for the FIA range of $0-180^{\circ}$, approximately isotropic systems had three positions to record sEMG signals under good conditions (MP was maximum). However, longitudinal and transversal highly anisotropic systems had two and one positions, respectively, at which sEMG signals were detected under good conditions. We showed also that the degree of isotropy of the nine detection systems investigated was less affected by the increase in muscle and fat thicknesses. However, with an increase in inter-electrode distance (IED), the degree of isotropy of approximately isotropic systems decreased while the degree of isotropy of highly anisotropic systems increased.
\end{abstract}

Keywords: Anisotropy, detection system, electrodes, electromyography, inclination.

Adv Biomed Eng. 7: pp. 107-116, 2018.

\section{Introduction}

The features of surface electromyographic (sEMG) signals are highly affected by anatomical, physiological, geometrical and detection system parameters [1,2]. Among the detection system parameters, the inclination of the electrodes relative to the orientation of muscle fibers can strongly influence the sEMG signal shape [3].

To better interpret electromyographic (EMG) signals generated from the same muscle of different subjects, the skin-fold thickness at the detection site and the orientation of electrodes with respect to the active muscle fibers must be taken into account [4].

For better recording of EMG signals, the electrode

Received on September 20, 2017; revised on January 2, 2018 and March 17, 2018; accepted on March 19, 2018.

* Department of Engineering of Electrical Systems, Faculty of Engineering Sciences, Université de Boumerdès, Boumerdès, Algeria.

** Department of Electronics, LIS Laboratory, Faculty of Technology, Université de Sétif 1, Sétif, Algeria.

\# Avenue de l'indépendance, 35000 Boumerdes, Algeria.

E-mail: messaoudinor@gmail.com orientation should be parallel to the fiber direction $[5,6]$. However, most muscles have pennate and/or curved muscle fibers, which imply that in the case of bipolar electrodes, for example, it is not always feasible to align the electrodes along the fiber direction [7]. Moreover, fibers within a muscle can be arranged in two major categories, parallel and pinnate. The fibers within a parallel muscle are approximately parallel to the long axis of the muscle fiber length [8] whereas the fibers within a pinnate muscle form an angle to the long axis of the muscle fiber length $[8,9]$. In addition, the detection system can also be inclined with respect to the muscle fiber direction $[1,10]$.

The sensitivity of a detection system to the muscle fiber orientation is related to the configuration of their electrodes. It has been shown that highly anisotropic, approximately isotropic [11,12] and isotropic systems [13] are very sensitive [12], less sensitive [11,12] and not sensitive [13], respectively, to the muscle fiber direction. A comparative study showed that amplitude and spectral variables of surface EMG signals are less affected by the fiber direction if almost isotropic detection systems are used than if anisotropic ones are used [1].

The sensitivities of the bi-transversal double differ- 
ential (BiTDD) [14] and the maximum kurtosis filter (MKF) [15] detection systems to fiber orientation have not been studied.

The location and orientation of the electrodes with respect to the muscle fiber direction were evaluated by amplitude and spectral contents of surface EMG signals [1,16-18]. Particularly, amplitude and mean power frequency (MPF) were used to examine the effects of electrode orientation $[1,16]$ on surface EMG signals. The effects of the skin fold thickness and the electrode orientation on the amplitude, degree of cross-correlation and median frequency (MDF) of surface EMG signals were examined to determine the good conditions for detecting surface EMG signal [4]. Zuniga et al. [16] studied the effects of two types of electrode orientation; parallel and perpendicular to the muscle fibers, on amplitude and MPF. However, the electrode orientation relative to the muscle fiber direction can be neither parallel nor perpendicular but in between the two [8,9].

The purpose of this study was to assess the effects of the inclination of muscle fibers with respect to the electrode orientation on the mean power of surface EMG signals detected by highly anisotropic and approximately isotropic systems.

This work was composed of two main parts. The first part was dedicated to the simulation of surface EMG signals generated in a multilayer cylindrical volume conductor consisting of the bone, muscle (the muscle layer includes a muscle of elliptical shape), fat and skin layers. The novelty of our simulation model resides mainly in its ability to simulate surface EMG signals generated in any geometry of the volume conductor (including cylindrical, planar and curvilinear). To simulate the sEMG signal generated in any geometry of a volume conductor, it is sufficient to introduce their parameters into the model function. The second part based on the first was devoted to the determination of the fiber inclination angles (FIAs) corresponding to the best orientation of the electrodes with respect to the direction of the muscle fibers in term of mean power (MP).

\section{Methods}

\subsection{Surface EMG signal simulation}

A model of surface EMG signal simulation was developed using MATLAB. Surface EMG signal simulation begins from simulation of the single fiber action potential (SFAP) [19], followed by simulation of the motor unit action potential (MUAP), which is the sum of the SFAPs belonging to the same motor unit (MU). After that, simulation of the MUAP train is based on repetition of the MUAP according to the motoneuron firing rate. Finally, surface EMG signal is the sum of the MUAP trains [20].

\subsubsection{Simulation of the SFAP}

Three major steps are necessary for SFAP modeling: 1 . description of the source; 2 . mathematical description of the volume conductor properties; and 3. modeling of the detection system [19].

The current density source is proportional to the second derivative of the intracellular action potential (IAP). This IAP was described analytically by Rosenfalck in 1969 [21]. For each muscle fiber, two IAPs are generated at the neuromuscular junction (NMJ), then they propagate in two opposite directions towards the tendons where they vanish [22].

We used a volume conductor described as multilayered cylinder composed of bone (homogeneous and isotropic, $20 \mathrm{~mm}$ in radius with conductivity set at $0.02 \mathrm{~S} / \mathrm{m}$ ), muscle (homogeneous and anisotropic, 26$\mathrm{mm}$ thick, with longitudinal conductivity set at $0.5 \mathrm{~S} / \mathrm{m}$ and transversal conductivity at $0.1 \mathrm{~S} / \mathrm{m}$ ), fat (homogeneous and isotropic, $3 \mathrm{~mm}$ thick, with conductivity set at $0.05 \mathrm{~S} / \mathrm{m}$ ) and skin (homogeneous and isotropic, $1 \mathrm{~mm}$ thick with conductivity set at $1 \mathrm{~S} / \mathrm{m}$. It was described analytically by a transfer function in the $2 \mathrm{D}$ spatial frequency domain [23].

The fiber density within the muscle was 20 fibers $/ \mathrm{mm}^{2}$ [24]. With this density, the number of fibers within the elliptical muscle was 171,270 (Table 1) $[24,26]$. The innervation zones were located at $50 \%$ of the length of the fibers and they had the same transverse position within the volume conductor [24]. The neuromuscular junctions and the ending tendons were uniformly distributed over a range of $5 \mathrm{~mm}$ [24].

In this study, the systems studied were divided into two groups: highly anisotropic and approximately isotropic. The highly anisotropic systems were the longitudinal single and double differential (LSD and LDD), and the transversal single and double differential (TSD and TDD) systems. The approximately isotropic systems

Table 1 Numerical details of the model (MFs = muscle fibers) [30].

\begin{tabular}{|c|c|c|c|c|}
\hline $\begin{array}{l}\text { MUs } \\
\text { diameters } \\
(\mathbf{m m})\end{array}$ & $\begin{array}{l}\text { MUs with } \\
\text { each } \\
\text { diameter }\end{array}$ & $\begin{array}{l}\text { MFs in } \\
\text { each MU }\end{array}$ & $\begin{array}{l}\text { MFs in } \\
\text { all MUs }\end{array}$ & $\begin{array}{l}\text { The } \\
\text { number of } \\
\text { MFs }\end{array}$ \\
\hline 2 & 28 & 63 & 1,764 & \multirow{7}{*}{$-171,270$} \\
\hline 3 & 50 & 141 & 7,050 & \\
\hline 4 & 40 & 251 & 10,040 & \\
\hline 5 & 67 & 393 & 26,331 & \\
\hline 6 & 54 & 565 & 30,510 & \\
\hline 7 & 68 & 770 & 52,360 & \\
\hline 8 & 43 & 1005 & 43,215 & \\
\hline
\end{tabular}


were the normal double differential (NDD), the inverse rectangle (IR), and the inverse binomial of order two (IB2) systems [11,12]. The bi-transversal double differential (BiTDD) [14] and the maximum kurtosis filter (MKF) [15] systems were also investigated. Radius of electrodes was $4 \mathrm{~mm}$. The inter-electrode distance was $20 \mathrm{~mm}$ in both longitudinal and transversal directions [24]. The fiber orientation with respect to electrode arrangement is shown in Fig. 1.

\subsubsection{Simulation of the MUAP and MUAP train}

The MUAP generated in each motor unit (MU) is the sum of the action potentials generated from the fibers belonging to this MU [19,22].

A muscle elliptical in shape composed of 350 motor units was simulated in the muscle layer. The cross section of the simulated ellipse had axes of 30 and $20 \mathrm{~mm}$. The MUs within the muscle were determined by uniformly selecting the $x-y$ coordinates of the centre of each MU territory within the elliptical cross section of the muscle (Fig. 2) [20,24,27]. The number of fibers within MUs was linked to their sizes. They were uniformly distributed in MU territories [24,27]. The diameters of MUs were distributed according to the Poisson law in the range of 2 to $8 \mathrm{~mm}$, with an average of $6 \mathrm{~mm}[25,27]$.

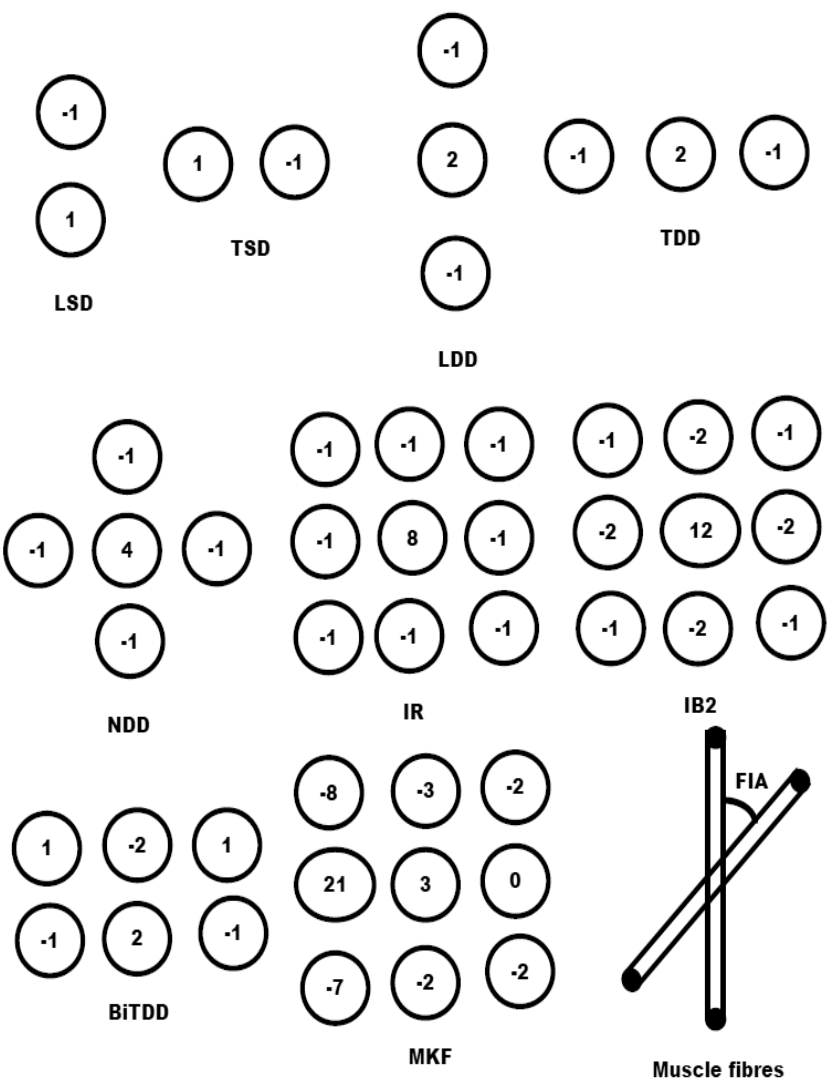

Fig. 1 Orientation of the electrodes configured in the investigated systems regarding the fiber direction.
Conduction velocities of all MUs were varied between $2.5 \mathrm{~m} / \mathrm{s}$ and $5.5 \mathrm{~m} / \mathrm{s}$. They were normally distributed in this interval with an average of $4 \mathrm{~m} / \mathrm{s}$ and standard deviation of $0.75 \mathrm{~m} / \mathrm{s}$ [27]. The motor unit action potential train (MUAPT) is the result of repeated discharge of an alpha-motor neuron [20]. The firing rates (FRs) of MUs ranged between $8 \mathrm{imp} / \mathrm{s}$ and $42 \mathrm{imp} / \mathrm{s}$. They were distributed on the activated MUs according to the Poisson law with an average of $12 \mathrm{imp} / \mathrm{s}$ [25]. According to the "size principle" rule, smaller motor units will be recruited at lower muscular voluntary contractions (MVCs) than larger ones. Moreover, the recruitment thresholds of motor units were distributed according to the Poisson law as the distribution of the motor unit's territories [20]. Therefore, the first recruited MU (smaller MU) had the largest firing rate, whereas the last recruited one (larger MU) had the smallest firing rate. Surface EMG signal is the sum of the resultant MUAPTs.

Figure 3 shows examples of simulated surface EMG signals detected by the LSD, BiTDD and MKF systems. The amplitude of the EMG signal detected by the MKF system was the largest and that amplitude of the EMG signal detected by the LSD system was the smallest.

The numerical details of the simulated model are shown in Table 1. Moreover, anatomical, physical and detection system parameters that were used to simulate surface EMG signals generated in a cylindrical multilayer volume conductor model are summarized in Table 2.

In clinical practice, the sEMG signals generated in a muscle is inevitably contaminated by various noise signals or artifacts that originate at the skin-electrode interface, in the electronics that amplifies the signals, and

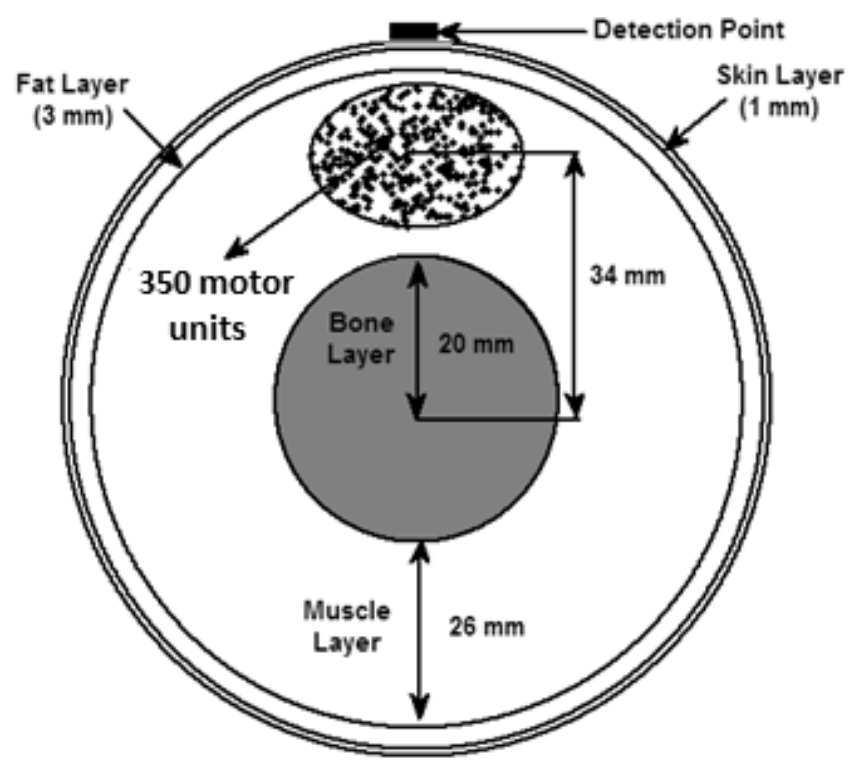

Fig. 2 The model of volume conductor used to simulate sEMG signals shown in Fig. 3 [29]. 


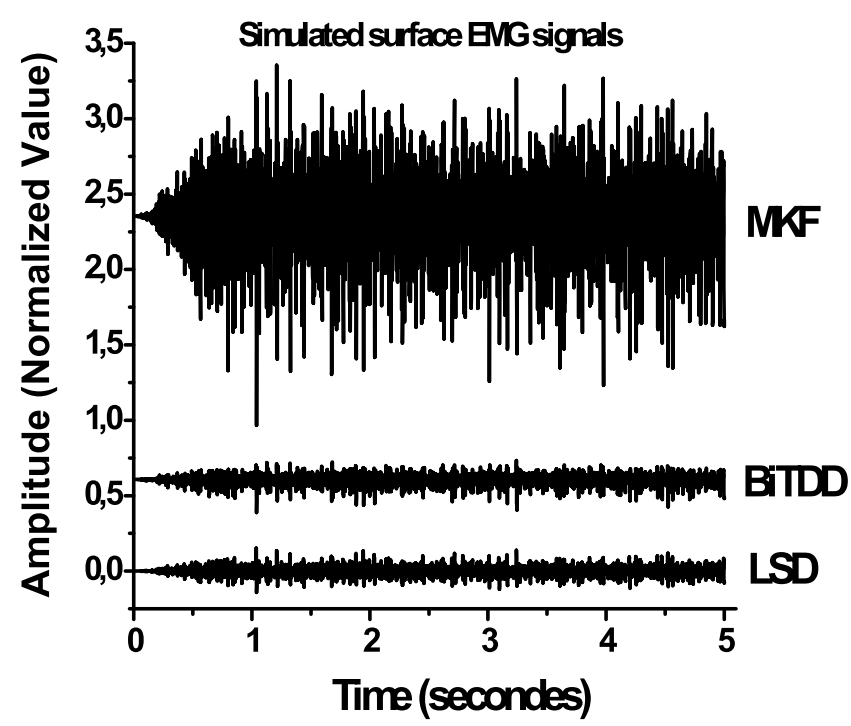

Fig. 3 Simulated sEMG signals generated in the volume conductor described in Fig. 2 and detected by LSD, BiTDD and MKF systems. The three signals were normalized with respect to the maximum amplitude of the MKF signal. Parameters of simulation are shown in Tables 1 and 2.

Table 2 The parameters selected to simulate sEMG signals [30].

\begin{tabular}{|c|c|c|}
\hline Parameter & Value & Description \\
\hline Fiber diameter & $46 \mu \mathrm{m}$ & The same for all fibers \\
\hline MFs lengths & 40 to $160 \mathrm{~mm}$ & $\begin{array}{l}\text { Gaussian distribution } \\
(\text { mean }=80 \mathrm{~mm}, \\
\mathrm{SD}=1 \mathrm{~mm})\end{array}$ \\
\hline MUs diameters & 2 to $8 \mathrm{~mm}$ & $\begin{array}{l}\text { Poisson distribution } \\
(\text { mean }=6 \mathrm{~mm})\end{array}$ \\
\hline MUs firing rates & 8 to $42 \mathrm{~Hz}$ & $\begin{array}{l}\text { Poisson distribution } \\
(\text { mean }=12 \mathrm{~Hz})\end{array}$ \\
\hline $\begin{array}{l}\text { MUs conduction } \\
\text { velocities }\end{array}$ & 2.5 to $5.5 \mathrm{~m} / \mathrm{s}$ & $\begin{array}{l}\text { Gaussian distribution } \\
(\text { mean }=4 \mathrm{~m} / \mathrm{s} \\
\mathrm{SD}=0.75 \mathrm{~m} / \mathrm{s})\end{array}$ \\
\hline MUs territory & - & Circular \\
\hline $\begin{array}{l}\text { MUs axis } \\
\text { distribution }\end{array}$ & - & Uniform distribution \\
\hline Number of MUs & 350 & \\
\hline $\begin{array}{l}\text { Fiber distribution } \\
\text { within the MUs }\end{array}$ & - & Uniform distribution \\
\hline Endplates spread & $5 \mathrm{~mm}$ & Uniform distribution \\
\hline $\begin{array}{l}\text { Tendons regions } \\
\text { spread }\end{array}$ & $5 \mathrm{~mm}$ & Uniform distribution \\
\hline $\begin{array}{l}\text { Muscle shape and } \\
\text { dimensions }\end{array}$ & $20 / 30 \mathrm{~mm}$ & Elliptic \\
\hline MFs density & 20 fibres $/ \mathrm{mm}^{2}$ & Of the muscle area \\
\hline Contraction Level & $70 \% \mathrm{MVC}$ & \\
\hline
\end{tabular}

from external sources [28]. Nevertheless, in our proposed model, examination of the effects of the element of artifacts on the detected sEMG signals was not included because the main objective of our study was not to implement these artifacts but to process and analyze the EMG signals detected by various systems in the case of inclined fibers.

\subsection{Estimation of the fibers inclination effect}

The objective of this study was to estimate the effects of fiber inclination with respect to the electrode orientation on the surface EMG signals. The fiber inclination with respect to the horizontal plane can be examined by considering the rotation of the detection system over the detection surface [9]. Here, the rotation of the detection system with respect to the muscle fiber direction in the horizontal plane was performed by varying the FIA from $0^{\circ}$ to $180^{\circ}$ in steps of $10^{\circ}$ (FIA is a parameter of the transfer function of the detection system as described in [19]). Then, the MP was calculated for each FIA. We found that the MPs of the signals detected by anisotropic and approximately isotropic detection systems are sensitive to the electrode misalignment. This sensitivity was different from one system to another. Therefore, we assumed that the FIA that matches the best orientation of the electrodes with respect to the muscle fiber direction is the one for which MP is maximum, and that the FIA corresponding to the worst orientation of the electrodes is the one for which MP is minimum.

Mean power is the average power of the EMG power spectrum. It is defined as follows [31]:

$$
M P=\left(\sum_{i=1}^{N} P_{i}\right) / N
$$

Where $N$ is the length of frequency bin and $P_{i}$ is the power spectrum of the simulated sEMG signal at frequency bin $i$.

To analyze the results of the MP and to classify the detection systems studied according to the degree of isotropy, the normalized difference between the maximum and the minimum mean power (NDMP) was used as a second parameter.

$$
N D M P=\left(M P_{\max }-M P_{\min }\right) / M P_{\max }
$$

The criterion for evaluating the degree of isotropy of detection systems is that the smaller the NDMP, the more isotropic is the detection system.

To take into consideration variations in the MPs computed with various initial conditions, the effects of thicknesses of muscle and fat layers and inter-electrode distances (IEDs) on the MPs of the sEMG signals detected by the nine systems were investigated. Three values were assigned to each of the parameters (Table 3). The evaluation of the effects of these three conditions on the 
degree of isotropy of the nine investigated detection systems is shown in Table 4.

\section{Results}

We evaluated the sensitivity of two categories of detection systems (approximately isotropic and very anisotropic) to muscle fiber orientation by computing the MPs of surface EMG signals detected by these recording systems as a function of the FIA.

The FIA has no effect on the MPs of sEMG signals detected by purely isotropic systems (ring systems and mono-polar system with circular electrode), since they are insensitive to the fiber orientation, $[1,7]$. On the basis of this property, we extended our analysis based on the MP to other systems used in surface EMG signal detection.

Figures 4-7 show the effects of the FIA on the MP of EMG signals detected by approximately isotropic (Fig. 4), highly anisotropic (Figs. 5 and 6), BiTDD and MKF (Fig. 7) systems for three muscle layer thicknesses. At each FIA, when the muscle layer thickness increased, the MPs of the sEMG signals detected by the nine systems decreased. The MP was also affected by the FIA, and the curve of the MP as a function of FIA differed from one detection system to another. This curve shows the behavior of each system with respect to the

Table 3 The values assigned to muscle and fat thicknesses and inter-electrode distance $[23,24]$.

\begin{tabular}{llll}
\hline Parameter & \multicolumn{3}{c}{ Value } \\
\hline Muscle thickness $(\mathbf{m m})$ & 24 & 26 & 28 \\
\hline Fat thickness $(\mathbf{m m})$ & 1 & 2 & 3 \\
\hline Inter-electrode distance $(\mathbf{m m})$ & 10 & 15 & 20 \\
\hline
\end{tabular}

fiber orientation. It can be considered as a response of the detection system to the fiber orientation.

As shown in Fig. 4, the curve of the IR system had four electrode orientations at which the MP was minimal, and the curves of the NDD and IB2 systems had only two positions at which the MP was minimal. However, the curves of these three systems had three positions at which the MP was maximal. Consequently, the approximately isotropic systems permit a very favorable detection of the EMG signals in three positions as specified by the $\mathrm{MP}_{\max }$.

As shown in Figs 5 and 6, the one-dimensional longitudinal and transversal detection systems in relation to the best electrode orientation behaved in a reverse manner. At FIA $=0^{\circ}$ and $180^{\circ}$, the LSD and LDD systems had the $\mathrm{MP}_{\max }$ while the TSD and TDD systems had the $\mathrm{MP}_{\text {min }}$. However, at FIA $=90^{\circ}$, the LSD and LDD systems had the $\mathrm{MP}_{\text {min }}$ while the TSD and TDD systems had the $\mathrm{MP}_{\max }$. Accordingly, for the FIA range of 0-180, longitudinal and transversal highly anisotropic detection systems had two and one electrode orientations, respectively, for good recording of EMG signals. This result implies that the detection systems of this category are very sensitive to the fiber orientation. Moreover, with this category of systems, the best position between the fiber orientation and the electrode arrangement is lost with a small angle of rotation of the detection system with respect to fiber orientation, and return to the best position requires a large rotation.

The behaviors of the BiTDD (Fig. 7a) and MKF (Fig. 7b) systems according to the fiber orientation have not been reported. This is the first time that these two systems are evaluated from the point of view of sensitivity to the muscle fiber orientation. The curve of the function $\mathrm{MP}=f(\mathrm{FIA})$ for the BiTDD $($ Fig. 7a) system had

Table 4 Classification of nine detection systems investigated according to the degree of isotropy for different muscle and fat layers thicknesses and inter-electrode distances.

\begin{tabular}{llllllllll}
\hline \multirow{2}{*}{$\begin{array}{c}\text { Detection } \\
\text { system }\end{array}$} & \multicolumn{9}{c}{ NDMP } \\
\cline { 2 - 11 } & \multicolumn{1}{c}{ Muscle thickness $(\mathbf{m m})$} & \multicolumn{7}{c}{ Fat thickness $(\mathbf{m m})$} & \multicolumn{3}{c}{ IED $(\mathbf{m m})$} \\
\cline { 2 - 10 } & $\mathbf{2 4}$ & $\mathbf{2 6}$ & $\mathbf{2 8}$ & $\mathbf{1}$ & $\mathbf{2}$ & $\mathbf{3}$ & $\mathbf{1 0}$ & $\mathbf{1 5}$ & $\mathbf{2 0}$ \\
\hline LSD & 0.6345 & 0.6362 & 0.6381 & 0.6362 & 0.6371 & 0.6380 & 0.7498 & 0.6757 & 0.6362 \\
\hline TSD & 0.6345 & 0.6362 & 0.6381 & 0.6362 & 0.6371 & 0.6380 & 0.7498 & 0.6757 & 0.6362 \\
\hline LDD & 0.5386 & 0.5414 & 0.5444 & 0.5414 & 0.5427 & 0.5442 & 0.6491 & 0.5891 & 0.5414 \\
\hline TDD & 0.5386 & 0.5414 & 0.5444 & 0.5414 & 0.5427 & 0.5442 & 0.6491 & 0.5891 & 0.5414 \\
\hline NDD & 0.0387 & 0.0374 & 0.0358 & 0.0374 & 0.0367 & 0.0359 & 0.0077 & 0.0163 & 0.0374 \\
\hline IR & 0.0044 & 0.0044 & 0.0045 & 0.0044 & 0.0044 & 0.0045 & 0.0035 & 0.0042 & 0.0044 \\
\hline IB2 & 0.0166 & 0.0164 & 0.0161 & 0.0164 & 0.0163 & 0.0161 & 0.0025 & 0.0054 & 0.0164 \\
\hline BiTDD & 0.4725 & 0.4759 & 0.4796 & 0.4759 & 0.4775 & 0.4795 & 0.4759 & 0.4759 & 0.4759 \\
\hline MKF & 0.7610 & 0.7574 & 0.7535 & 0.7574 & 0.7557 & 0.7536 & 0.5129 & 0.6564 & 0.7574 \\
\hline
\end{tabular}



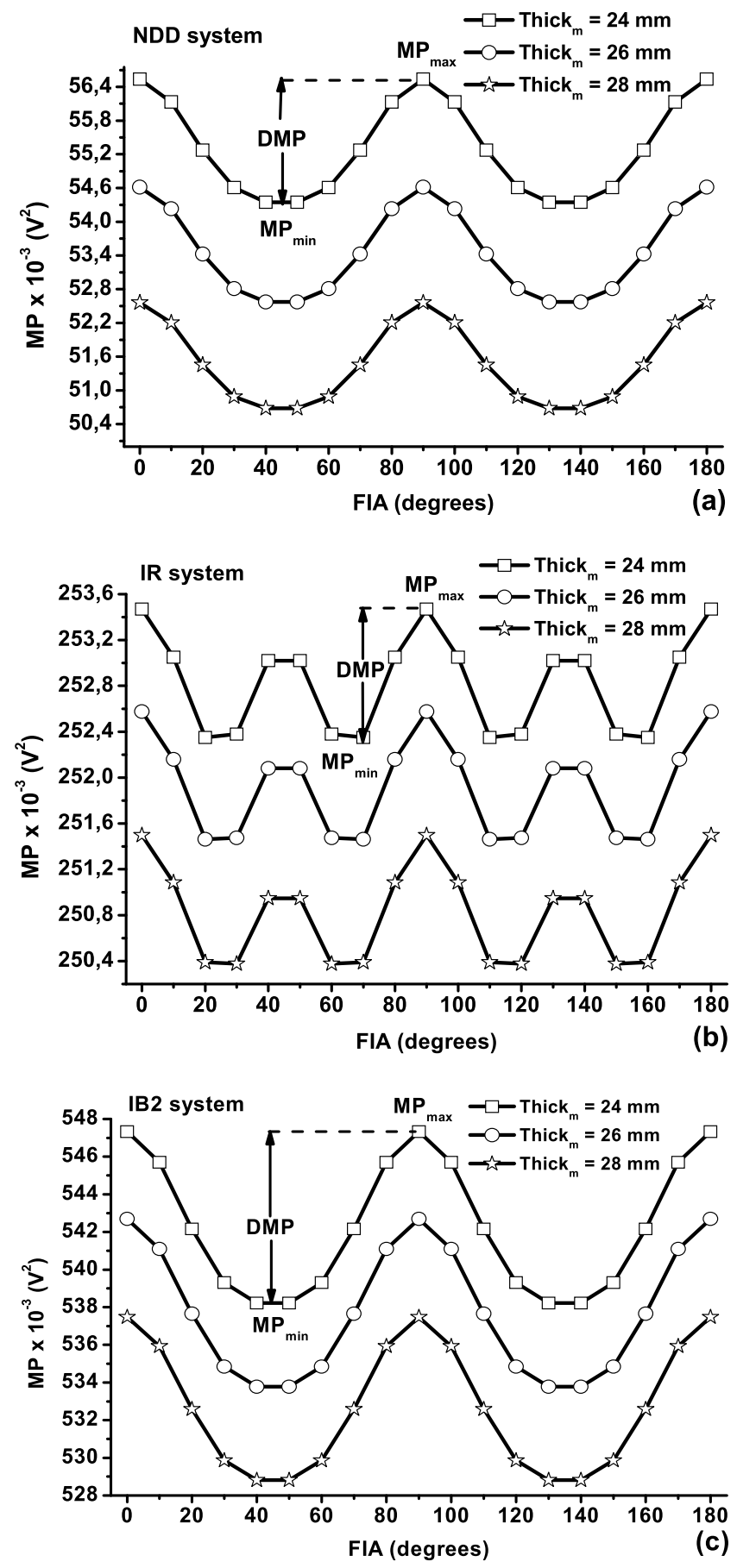

Fig. 4 Influence of FIA on MPs of sEMG signals detected by the approximately isotropic systems for three muscle layer thicknesses $\left(\right.$ Thick $\left._{\mathrm{m}}\right)$.

one position (at FIA $=90^{\circ}$ ) corresponding to the best electrode orientation and two positions (at FIA $=30^{\circ}$ and FIA $=150^{\circ}$ ) corresponding to the worst electrode orientation. However, the curve of the MKF system (Fig. 7b) had one position (at FIA $=90^{\circ}$ ) corresponding to the best electrode orientation and one position (at FIA $=160^{\circ}$ ) corresponding to the worst orientation.

Table 4 presents the NDMP results as a function of muscle thickness, fat thickness, and IED for the nine sys-
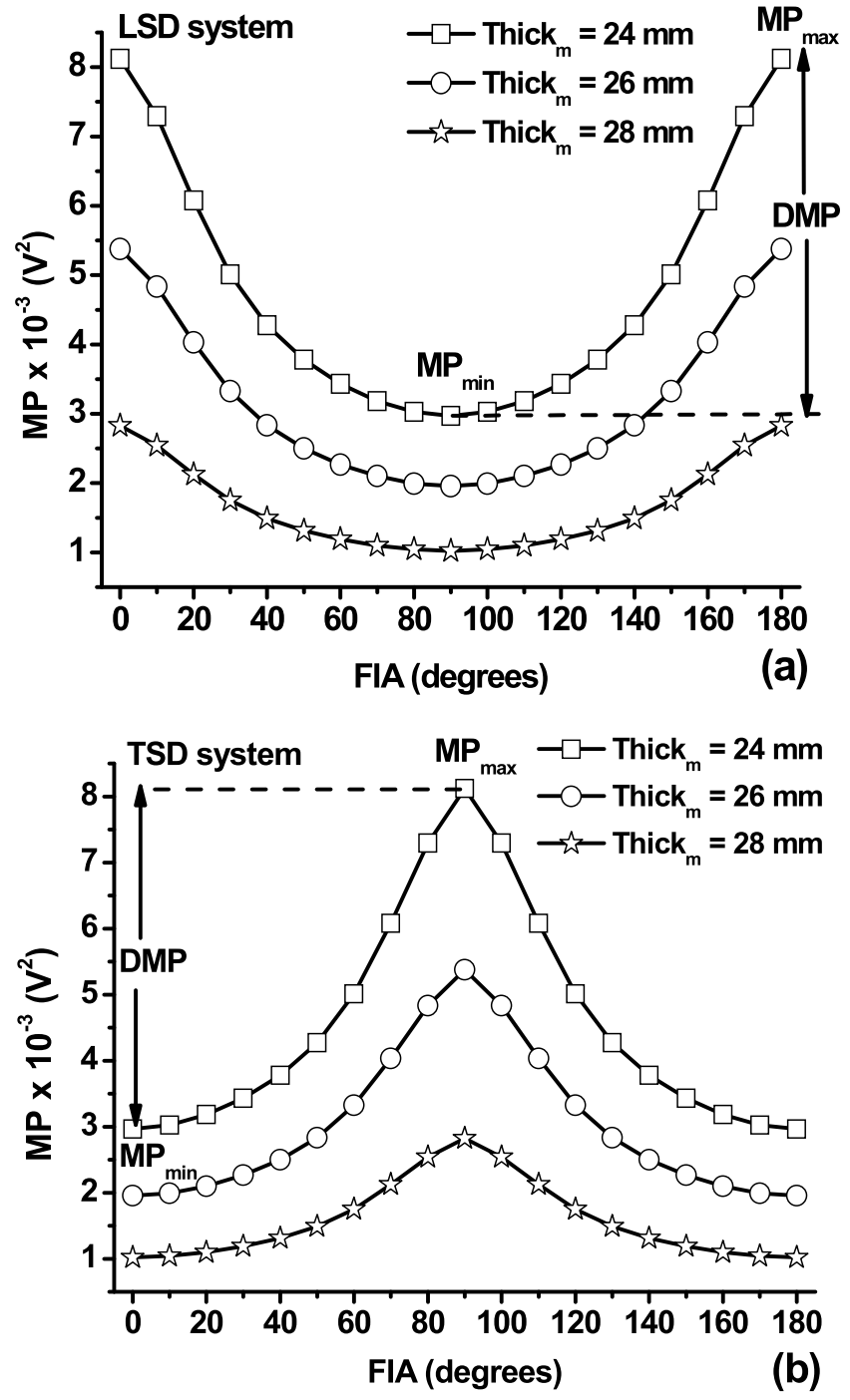

Fig. 5 Influence of FIA on MPs of sEMG signals detected by the LSD and TSD detection systems for three muscle layer thicknesses ( Thick $_{\mathrm{m}}$ ).

tems studied. The degree of isotropy of a detection system was not only related to the system itself but also to other parameters such as the thicknesses of the muscle and fat layers and the IED. The findings further suggest that:

- Although the MKF system is a 2D system but it is the most anisotropic system, except when IED $=10$ and $15 \mathrm{~mm}$ at which the LSD system is the most anisotropic.

- The IR system is the most isotropic system, except when IED $=10 \mathrm{~mm}$ at which the IB2 system is the most isotropic system.

- The degree of isotropy of the nine detection systems studied is more influenced by the variation of IED than by the variation of muscle and fat thicknesses. Particularly, the degree of isotropy of the BiTDD system is not influenced by variation of IED. 

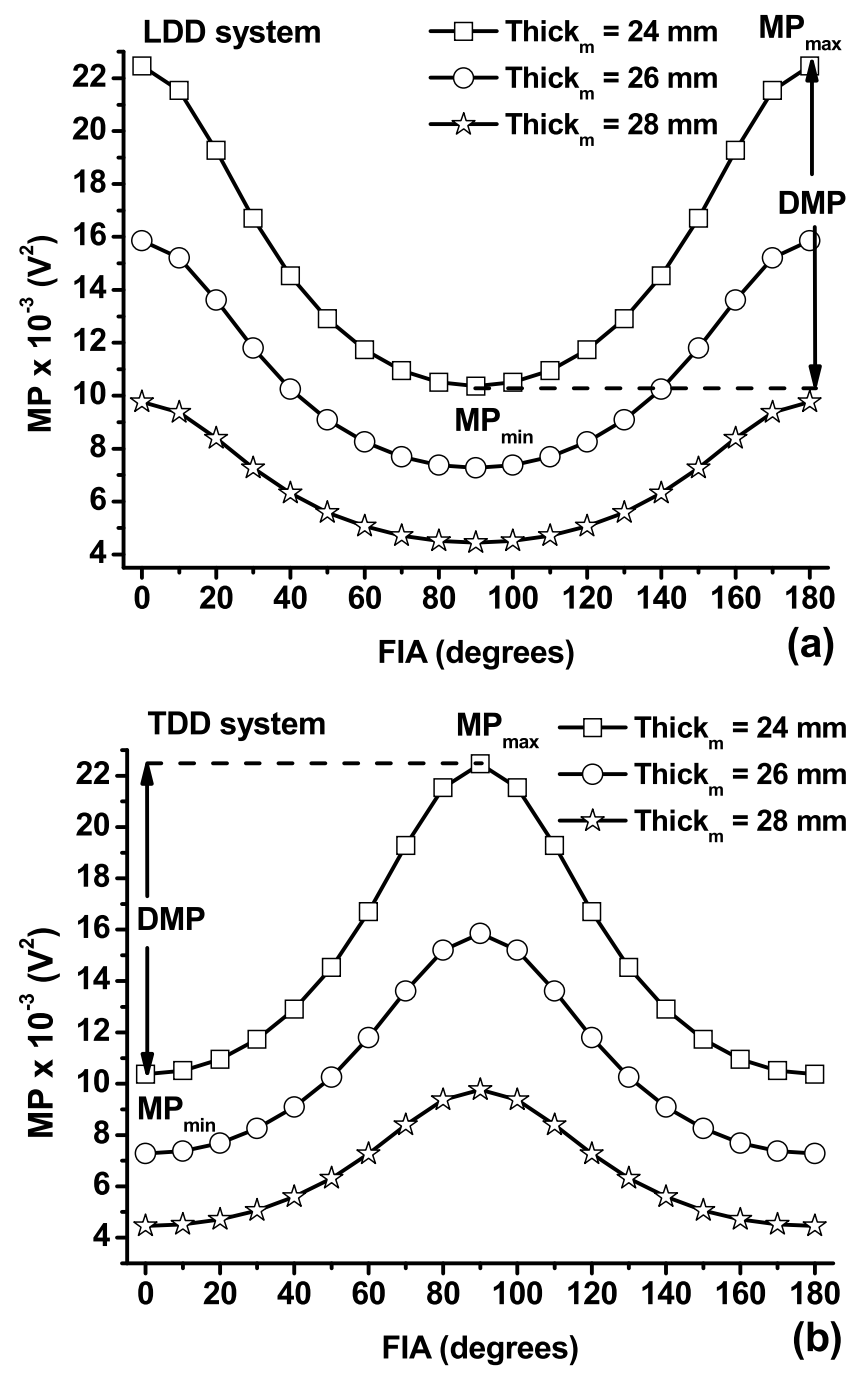

Fig. 6 Influence of FIA on MPs of sEMG signals detected by the LDD and TDD detection systems for three muscle layer thicknesses $\left(\right.$ Thick $\left._{\mathrm{m}}\right)$.

- When the IED increases, the NDMP decreases in very anisotropic systems (LSD, LDD, TSD and TDD) and increases in approximately isotropic systems (NDD, IR and IB2). According to the criterion for evaluating the degree of isotropy of detection systems, the increase in IED leads to an increase in degree of anisotropy in the very anisotropic systems and to a decrease in degree of anisotropy in the almost isotropic systems.

\section{Discussion}

The power spectrum has been widely used to analyze the effect of anatomical, physical and detection system parameters on EMG signals [32, 33]. Particularly, the location of the electrodes along an active muscle fiber has been shown to affect the power spectrum of the action potential generated from the muscle fiber [32]. Since the MP is the average power spectrum [31], the effect of
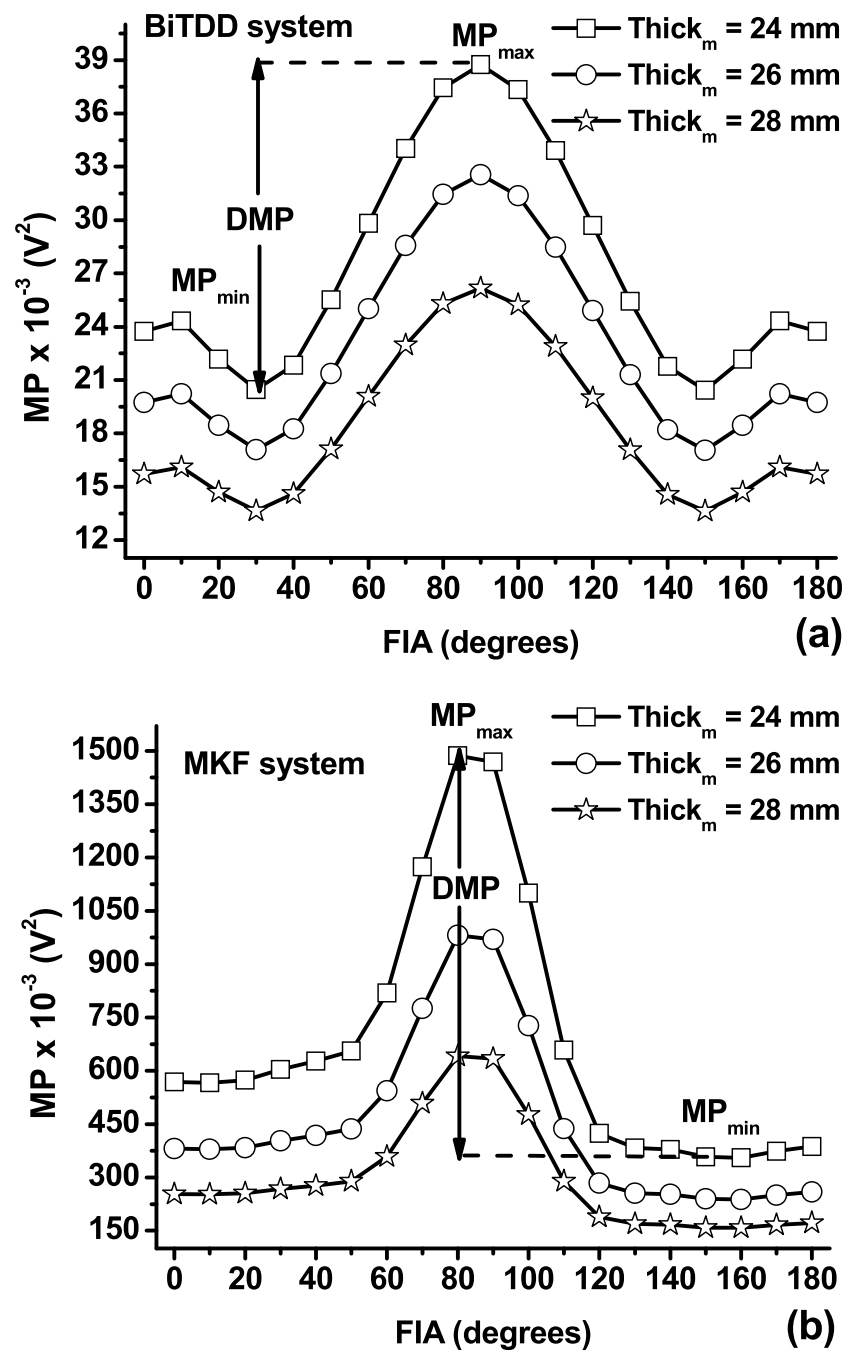

Fig. 7 Effect of FIA on MPs of sEMG signals detected by the BiTDD and the MKF systems for three muscle layer thicknesses $\left(\right.$ Thick $\left._{\mathrm{m}}\right)$.

each anatomical or physical or detection system parameter on the power spectrum of the detected surface EMG signals can be evaluated by the MP. Therefore, we used the MP of the surface EMG signal as a parameter to estimate the effect of fiber inclination with respect to the electrode orientation on the detected surface EMG signal. Our focus was to observe the changes in this parameter when the orientation of the electrodes was modified with respect to the direction of muscle fibers.

At FIA ranging from $0^{\circ}$ to $180^{\circ}$, longitudinal and transversal highly anisotropic detection systems have two and one orientations, respectively, with respect to the direction of muscle fibers where MP is maximum (Figs. 5 and 6), and the approximately isotropic detection systems have three orientations (Fig. 4).

The sensitivity of anisotropic and approximately isotropic detection systems to fiber orientation has been assessed by amplitude and spectral content of the 
SFAP [1]. The behaviors of the isotropic detection systems as a function of the fiber orientation were also examined [13]. However, the sensitivity of the BiTDD [14] and the MKF [15] systems to the muscle fiber orientation has never been examined. In addition, there is no study that determines FIAs corresponding to the best (or worst) orientation of the electrodes of highly anisotropic and approximately isotropic systems with respect to the direction of muscle fibers to ensure proper detection of surface EMG signal. Moreover, assessment of the degree of isotropy and/or anisotropy of detection systems has never been studied. In this study, the degree of isotropy of approximately isotropic and very anisotropic systems as a function of muscle and fat layers thicknesses and inter-electrode distances was assessed as shown in Table 4. The results show that the degree of isotropy and/or anisotropy of a detection system is not only related to the configuration of their electrodes with respect to the muscle fiber orientation but also to other parameters such as the thicknesses of muscle and fat layers and IED.

It is noteworthy that approximately isotropic detection systems are less sensitive to the muscle fiber orientation compared to the highly anisotropic systems. Therefore, the amplitude and spectral contents of SFAP are less affected by the orientation of approximately isotropic detection systems [1]. This effect was observed in our results by the NDMP (Table 4). Since the NDMP of the signals detected by the isotropic detection systems is zero, we have established that the smaller the NDMP of a system, the greater is the isotropy (Table 4).

Focusing on the approximately isotropic systems, these detection systems do not have the same sensitivity to the muscle fiber orientation $[1,11]$. Theoretically, Disselhorst-Klug et al. [11] mentioned that the transfer function of the IR system showed higher isotropy compared to the NDD system transfer function, and that the IB2 transfer function showed higher isotropy compared to the IR transfer function. In this work, the behaviors of this category of systems as a function of FIA were evaluated by the NDMP. We found that the NDMP for the IR system is always inferior to the NDMP for the IB2 system and that the NDMP for the IB2 system is inferior to the NDMP for the NDD system, with the exception when IED $=10 \mathrm{~mm}$ (Table 4). Therefore, for these three systems, the result of Disselhorst-Klug et al. [11] was confirmed by using NDMP only for IED $=10 \mathrm{~mm}$. This implies that the degree of isotropy of a detection system may be influenced by other parameters. Moreover, we showed that the number of FIAs corresponding to the best $\left(\mathrm{MP}_{\max }\right)$ and worst $\left(\mathrm{MP}_{\min }\right)$ electrode orientations for the IR (Figs. 4b) system is different from those of the NDD and IB2 systems (Figs. 4a and 4c). Focusing on the number of FIAs corresponding to the best and the worst positions of the electrodes with respect to fiber orientation in the case of approximately detection systems, when the FIA varied from $0^{\circ}$ to $180^{\circ}$ in steps of $10^{\circ}$, the best $\left(\mathrm{MP}_{\max }\right)$ orientation of the electrodes with respect to the muscle fiber direction was at FIA of $90^{\circ}$ for the NDD (Fig. 4a), IR (Fig. 4b) and IB2 (Fig. 4c) systems. However, the worst $\left(\mathrm{MP}_{\min }\right)$ orientation was at FIA of $45^{\circ}$ and at FIA of $135^{\circ}$ for the NDD (Fig. 4a) and IB2 (Fig. 4c) systems and in permutation between $20^{\circ}, 70^{\circ}, 110^{\circ}, 160^{\circ}$ for the IR system.

Dimitrov et al. [14] showed in a simulation study that the BiTDD system was more selective than the LDD, TDD and NDD systems and promised the strongest reduction of cross-talk. However, the behaviors of these four systems with respect to the muscle fiber direction were not investigated in their work. In this study, we showed that the BiTDD system is highly anisotropic and is the least sensitive to the orientation of muscle fibers compared to the four other systems. In fact, Table 4 shows that the NDMP of the BiTDD system was smaller than the NDMPs of the LSD, TSD, LDD and TDD systems.

Östlund et al. [15] showed that the MKF system was better at enhancing a single MUAP than highly anisotropic and approximately isotropic detection systems for all levels of spatial correlation of the noise. However, the sensitivity of the orientation of electrodes of this system to the muscle fiber direction has not been studied. We showed in Table 4 that the MKF system is highly anisotropic and is the most sensitive to the muscle fiber direction compared to the other systems investigated, with the exception when IED $=10 \mathrm{~mm}$ and IED $=15 \mathrm{~mm}$ at which the LSD, TSD, LDD and TDD systems are more anisotropic compared to the MKF system. Thus, the anisotropy of the BiTDD and the MKF systems, which had not been defined until now, was determined in this work, and BiTDD and MKF systems can be considered as highly anisotropic detection systems.

\section{Conclusions}

A major outcome of this study is a recommendation based on the mean power for the estimation of the effect of fiber inclination on the detected surface EMG signals. As a result, $\mathrm{MP}_{\max }$ indicates the best possible position of the electrode orientation with respect to fiber direction and $\mathrm{MP}_{\min }$ indicates the worst position.

Our results show that we can:

Determine the best and the worst orientation of the electrodes relative to the muscle fiber direction for any detection system.

Compare the degree of isotropy of detection systems using the NDMP. Thus, the smaller the NDMP of a system, the greater is the isotropy. 
In addition, the behaviors of the BiTDD and MKF systems as a function of the fiber inclination were investigated for the first time in this study and compared with highly anisotropic and approximately isotropic systems. Finally, we categorize the BiTDD and the MKF systems as highly anisotropic systems and show that the degree of isotropy of a detection system can be influenced by other factors such as muscle and fat thicknesses and inter-electrode distances.

\section{References}

1. Farina D, Cescon C, Merletti R: Influence of anatomical, physi$\mathrm{cal}$, and detection-system parameters on surface EMG. Biol Cybern. 86(6), pp. 445-456, 2002.

2. Farina D, Merletti R, Enoka RM: The extraction of neural strategies from the surface EMG. J Appl Physiol. 96(4), pp. 14861495, 2004.

3. Mesin L, Merletti R, Rainoldi A: Surface EMG: the issue of electrode location. J Electromyogr Kinesiol. 19(5), pp. 719-726, 2009.

4. De la Barrera EJ, Milner TE: The effects of skinfold thickness on the selectivity of surface EMG. Electroencephalogr. Clinic Neurophysiol. 93(2), pp. 91-99, 1994.

5. $\mathrm{Ng} \mathrm{JK}$, Kippers V, Richardson CA: Muscle fibre orientation of abdominal muscles and suggested surface EMG electrode positions. Electroencephalogr. Clinic Neurophysiol. 38(1), pp. 51$58,1998$.

6. Hermens HJ, Freriks B, Disselhorst-Klug C, Rau G: Development of recommendations for SEMG sensors and sensor placement procedures. J Electromyogr Kinesiol. 10(5), pp. 361-374, 2000.

7. Teklemariam A, Hodson-Tole EF, Reeves ND, Costen NP, Cooper G: A Finite Element Model Approach to Determine the Influence of Electrode Design and Muscle Architecture on Myoelectric Signal Properties. PLoS ONE. 11(2): e0148275. doi:10.1371/ journal.pone.0148275, 2016.

8. Fukunaga T, Kawakami Y, Kuno S: Muscle architecture and function in humans. J Biomech. 30(5), pp. 457-463, 1997.

9. Mesin L, Farina D: Simulation of surface EMG signals generated by muscle tissues with inhomegeneity due to fiber pennation. IEEE Trans Biomed Eng. 51(9), pp. 1521-1529, 2004.

10. Dimitrova NA, Dimitrov AG, Dimitrov GV: Calculation of extracellular potentials produced by an inclined muscle fibre at a rectangular plate electrode. Med Eng Phy. 21(8), pp. 583-588, 1999.

11. Disselhorst-Klug C, Silny J, Rau G: Improvement of spatial resolution in surface-EMG: A theoretical and experimental comparison of different spatial filters. IEEE Trans Biomed Eng. 44(7), pp. 567-574, 1997.

12. Farina D, Arendt-Nielsen L, Merletti R, Indino B, Graven-Nielsen T: Selectivity of spatial filters for surface EMG detection from the tibialis anterior muscle. IEEE Trans Biomed Eng. 50(3), pp. 354-364, 2003.

13. Farina D, Cescon C: Concentric-ring electrode systems for noninvasive detection of single motor unit activity', IEEE Trans Biomed Eng. 48(11), pp. 1326-1334, 2001.

14. Dimitrov GV, Disselhorst-Klug C, Dimitrova NA, Schulte E, Rau G: Simulation analysis of the ability of different types of multi-electrodes to increase selectivity of detection and to reduce cross-talk. J Electromyogr Kinesiol. 13(2), pp. 125-138, 2003.

15. Östlund N, Yu J, Roeleveld K, Karlsson JS: Adaptive spatial filtering of multichannel surface electromyogram signals. Med Biol Eng Comput. 42(6), pp. 825-831, 2004.

16. Zuniga JM, Housh TJ, Hendrix CR, Camic CL, Mielke M, Schmidt RJ, Johnson GO: The effects of electrode orientation on electromyographic amplitude and mean power frequency during cycle ergometry. J Neurosc Meth. 184(2), pp. 256-262, 2009.

17. Beck TW, Housh TJ, Cramer JT, Weir JP: The effects of inter-electrode distance over the innervation zone and normalization on the electromyographic amplitude and mean power frequency versus concentric, eccentric, and isometric torque relationships for the vastus lateralis muscle. J Electromyogr Kinesiol. 19(2), pp. 219-231, 2009.

18. Beck TW, Housh TJ, Cramer and JT, Weir, JP: The effects of electrode placement and innervation zone location on the electromyographic amplitude and mean power frequency versus-isometric torque relationships for the vastus lateralis muscle. J Electromyogr Kinesiol.18(2), pp. 317-328, 2008.

19. Farina D, Merletti R: A novel approache for precise simulation of the EMG signal detected by surface electrodes. IEEE Trans Biomed Eng. 48(6), pp. 637-646, 2001.

20. Stashuk DW: Simulation of electromyographic signals. J Electromyogr Kinesiol. 3(3), pp. 157-173, 1993.

21. Rosenfalck P: Intra and extracellular fields of active nerve and muscle fibres: A physico-mathematical analysis of different models. Acta Physiol Scand Suppl. 321, pp. 1-168, 1969.

22. Dimitrov GV, Dimitrova NA: Precise and fast calculation of the motor unit potentials detected by a point and rectangular plate electrode. Med Eng Phy. 20(5), pp. 374-381, (1998).

23. Farina D, Mesin L, Simone M, Merletti R: A surface EMG generation model with multilayer cylindrical description of the volume conductor. IEEE Trans Biomed Eng. 51(3), pp. 415-426, 2004.

24. Keenan KG, Farina D, Meyer FG, Merletti R, Enoka RM: Sensitivity of the cross-correlation between simulated surface EMGs for two muscles to detect motor unit synchronization. J Appl Physiol. 102(3), pp. 1193-1201, 2007.

25. Wang W, Stefano Ade, Allen R: A simulation model of the surface EMG signal for analysis of muscle activity during the gait cycle. Comput Biol Med. 36(6), pp. 601-618, 2006.

26. Keenan KG, Farina D, Meyer FG, Merletti R, Enoka RM: Experimentally Valid Predictions of Muscle Force and EMG in Models of Motor-Unit Function Are Most Sensitive to Neural Properties. J Neurophysiol. 98(7), pp. 1581-1590, 2007.

27. Fuglevand AJ, Winter DA, Patla AE: Models of recruitment and rate coding organisation in motor-unit pools. J Neurophysiol. 70(6), pp. 2470-2488, 1993.

28. De Luca CJ, Donald GL, Kuznetsov M, Roy SH: Filtering the surface EMG signal: Movement artifact and baseline noise contamination. J Biomech. 43(8), pp. 1573-1579, 2010.

29. N. Messaoudi, R. E. Bekka: From Single Fibre Action Potential to Surface Electromyographic Signal: A Simulation Study. Third International Work Conference, IWBBIO 2015, Granada, Spain, Proceedings, Part I, LNCS 9043, April 15-17, 2015, pp. 315324, 2015.

30. Messaoudi N, Bekka RE, Ravier P, Harba R: Assessment of the 
non-Gaussianity and non-linearity levels of simulated sEMG signals on stationary segments. J Electromyog Kinesiol. 32(1), pp. 70-82, 2017.

31. Phinyomark A, Phukpattaranont P, Limsakul C: Feature reduction and selection for EMG signal classification. Expert Syst Appl. 39(8), pp. 7420-7431, 2012.

32. Lateva ZC, Dimitrova NA, Dimitrov GV: Effect of recording electrode position along a muscle fiber on surface potential power spectrum. J Electromyogr Kinesiol. 3(4), pp. 195-204, 1993.

33. Dimitrov GV, Dimitrova NA: Fundamentals of power spectra of extracellular potentials produced by a skeletal muscle fibre of finite length Part I: Effect of fibre anatomy. Med Eng Phy. 20 (8), pp. 580-587, 1998.

\section{Noureddine MESSAOUdI}

Dr. Noureddine Messaoudi graduated in electronics engineering from the University of Sétif $1, \mathrm{Al}$ geria, 2003. He received his Magister degree and his $\mathrm{PhD}$ degree in Electronics from the University of Sétif 1, Algeria, in 2006 and 2017, respectively. From 2008 until now, he is an assistant professor at

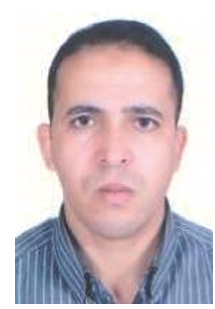
the University of Boumerdes where he teaches courses in Biomedical Engineering and Telecommunications. His main research interests concern surface EMG signals modeling and processing.

\section{Raïs El'hadi BEKKA}

Prof. Raïs El'hadi BeKKA graduated in Electronics Engineering from the Ecole Polytechnique, Alger, 1980. He received his Magister degree and the Doctorat d'Etat degree in Electronics from the University of Sétif 1, Algeria, in 1987 and 1994, respectively. Since May 2000, he is Professor at University of Sétif 1. His research interests include signal processing, modelling of surface EMG signals and multiple-description image coding.

\section{Samia Belkacem}

Dr. Samia BelKaCem graduated in electronics engineering from the University of Batna, Algeria in 2001. She received his Magister degree and his $\mathrm{PhD}$ degree in Electronics from the University of Batna, Algeria, in 2005 and 2015, respectively. In 2008, she joined the University of Boumerdes and holds the position of assistant professor. Her main research interests are in physiological signals and image processing.
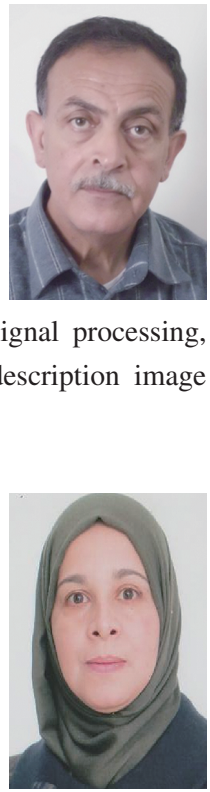